\title{
Wavelet Based Image Denoising Technique
}

\author{
Sachin D Ruikar \\ Department of Electronics and Telecommunication \\ Engineering \\ Shri Guru Gobind Singhji Institute of Engineering and \\ Technology, \\ Nanded, India \\ ruikarsachin@gmail.com
}

\author{
Dharmpal D Doye \\ Department of Electronics and Telecommunication \\ Engineering \\ Shri Guru Gobind Singhji Institute of Engineering and \\ Technology, \\ Nanded, India \\ dddoye@yahoo.com
}

\begin{abstract}
This paper proposes different approaches of wavelet based image denoising methods. The search for efficient image denoising methods is still a valid challenge at the crossing of functional analysis and statistics. In spite of the sophistication of the recently proposed methods, most algorithms have not yet attained a desirable level of applicability. Wavelet algorithms are useful tool for signal processing such as image compression and denoising. Multi wavelets can be considered as an extension of scalar wavelets. The main aim is to modify the wavelet coefficients in the new basis, the noise can be removed from the data. In this paper, we extend the existing technique and providing a comprehensive evaluation of the proposed method. Results based on different noise, such as Gaussian, Poisson's, Salt and Pepper, and Speckle performed in this paper. A signal to noise ratio as a measure of the quality of denoising was preferred.
\end{abstract}

Keywords: Image; Denoising; Wavelet Transform; Signal to Noise ratio; Kernel;

\section{INTRODUCTION}

The image usually has noise which is not easily eliminated in image processing. According to actual image characteristic, noise statistical property and frequency spectrum distribution rule, people have developed many methods of eliminating noises, which approximately are divided into space and transformation fields The space field is data operation carried on the original image, and processes the image grey value, like neighborhood average method, wiener filter, center value filter and so on. The transformation field is management in the transformation field of images, and the coefficients after transformation are processed. Then the aim of eliminating noise is achieved by inverse transformation, like wavelet transform [1], [2]. Successful exploitation of wavelet transform might lessen the noise effect or even overcome it completely [3]. There are two main types of wavelet transform - continuous and discrete [2]. Because of computers discrete nature, computer programs use the discrete wavelet transform. The discrete transform is very efficient from the computational point of view. In this paper, we will mostly deal with the modeling of the wavelet transform coefficients of natural images and its application to the image denoising problem. The denoising of a natural image corrupted by Gaussian noise is a classic problem in signal processing [4]. The wavelet transform has become an important tool for this problem due to its energy compaction property [5]. Indeed, wavelets provide a framework for signal decomposition in the form of a sequence of signals known as approximation signals with decreasing resolution supplemented by a sequence of additional touches called details [6][7]. Denoising or estimation of functions, involves reconstituting the signal as well as possible on the basis of the observations of a useful signal corrupted by noise [8] [9] [10] [11]. The methods based on wavelet representations yield very simple algorithms that are often more powerful and easy to work with than traditional methods of function estimation [12]. It consists of decomposing the observed signal into wavelets and using thresholds to select the coefficients, from which a signal is synthesized [5]. Image denoising algorithm consists of few steps; consider an input signal $\mathbf{x}(\mathbf{t})$ and noisy signal $\mathbf{n}(\mathbf{t})$. Add these components to get noisy data $\mathbf{y}(\mathbf{t})$ i.e.

$$
\mathbf{y}(\mathbf{t})=\mathbf{x}(\mathbf{t})+\mathbf{n}(\mathbf{t}) .
$$

Here the noise can be Gaussian, Poisson's, speckle and Salt and pepper, then apply wavelet transform to get $\mathbf{w}(\mathbf{t})$.

$$
\mathbf{y}(\mathbf{t}) \stackrel{\text { Wavelet Transform }}{\longrightarrow} \mathbf{w}(\mathbf{t}) \text {. }
$$

Modify the wavelet coefficient $\mathbf{w}(\mathbf{t})$ using different threshold algorithm and take inverse wavelet transform to get denoising image $\hat{\mathbf{x}}(\mathbf{t})$.

$$
\mathbf{w}(\mathbf{t}) \stackrel{\text { Inverse Wavelet Transform }}{\longrightarrow} \hat{\mathbf{x}}(\mathbf{t}) .
$$

The system is expressed in Fig. 1.

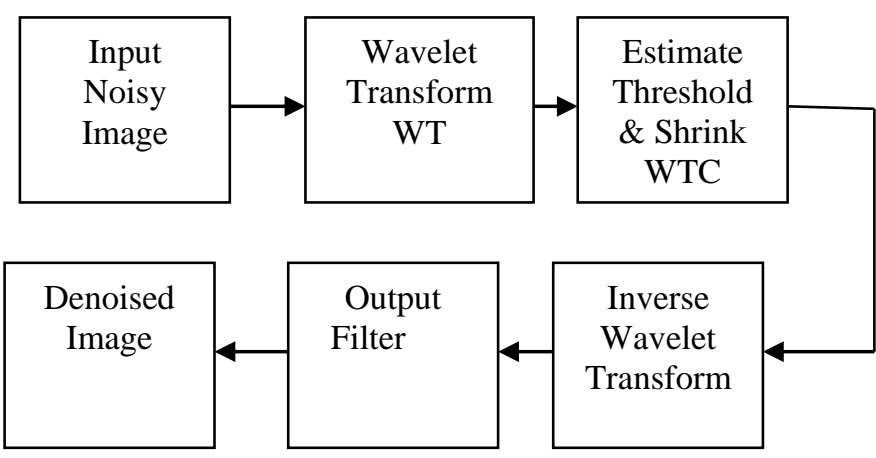

Figure 1: Block diagram of Image denoising using wavelet transform.

Image quality was expressed using signal to noise ratio of denoised image.

\section{WAVELET TRANSFORM}

The wavelet expansion set is not unique. A wavelet system is a set of building blocks to construct or represents a signal or function. It is a two dimensional expansion set, usually a basis, for some class one or higher dimensional signals. The wavelet expansion gives a time frequency localization of the signal. Wavelet systems are generated from single scaling function by 
scaling and translation. A set of scaling function in terms of integer translates of the basic scaling function by

$$
\boldsymbol{\varphi}_{\mathbf{k}}(\mathrm{t})=\boldsymbol{\varphi}(\mathbf{t}-\mathbf{k}) \quad \mathbf{k} \in \mathbf{Z} \quad \boldsymbol{\varphi} \in \mathbf{L}^{2} .
$$

The subspaces of $L^{2}(R)$ spanned by these functions is defined as $\mathbf{v}_{\mathbf{0}}={ }_{\mathbf{k}}^{\overline{\mathbf{S p a n}}_{\mathbf{k}}}\left\{\boldsymbol{\varphi}_{\mathbf{k}}(\mathbf{t})\right\}$ for all integers $\mathrm{k}$ from minus infinity to infinity. A two dimensional function is generated from the basic scaling function by scaling and translation by

$$
\boldsymbol{\varphi}_{\mathrm{j}, \mathrm{k}}(\mathrm{t})=\mathbf{2}^{\mathrm{j} / 2} \boldsymbol{\varphi}\left(2^{\mathrm{j}} \mathrm{t}-\mathbf{k}\right)
$$

Whose span over $\mathrm{k}$ is

$$
\mathbf{v}_{\mathbf{j}}={ }_{\mathbf{k}}^{\overline{\operatorname{Span}_{1}}}\left\{\boldsymbol{\varphi}_{\mathbf{k}}\left(2^{\mathbf{j}} \mathbf{t}\right)\right\}=\mathbf{v}_{\mathbf{j}}={ }_{\mathbf{k}}^{\overline{\operatorname{Span}}}\left\{\varphi_{\mathbf{j}, \mathbf{k}}(\mathbf{t})\right\}, \mathbf{k} \in \mathbf{Z} .
$$

for all integer. The multiresolution analysis expressed in terms of the nesting of spanned spaces as........ $\subset \mathbf{v}_{-2} \subset \mathbf{v}_{-1} \subset \mathbf{v}_{\mathbf{0}} \subset$ $\mathbf{v}_{\mathbf{1}} \subset \mathbf{v}_{\mathbf{2}} \subset \cdots . \subset \mathbf{L}^{2}$. The spaces that contain high resolution signals will contain those of lower resolution also. The spaces should satisfy natural scaling condition $\mathbf{f}(\mathbf{t}) \in \mathbf{v}_{\mathbf{j}} \Leftrightarrow \mathbf{f}(\mathbf{2 t}) \in$ $\mathbf{v}_{\mathbf{j}+\mathbf{1}}$ which ensures elements in space are simply scaled version of the next space. The nesting of the spans of $\boldsymbol{\varphi}\left(2^{\mathbf{j}} \mathbf{t}-\right.$ $\mathbf{k}$ )denoted by $\mathbf{v}_{\mathbf{j}}$ i.e. $\boldsymbol{\varphi ( t )}$ is in $\mathbf{v}_{\mathbf{0}}$, it is also in $\mathbf{v}_{\mathbf{1}}$, the space spanned by $\boldsymbol{\varphi ( 2 t )}$. This $\boldsymbol{\varphi ( t )}$ can be expressed in weighted sum of shifted $\boldsymbol{\varphi ( 2 t ) \text { as }}$

$$
\boldsymbol{\varphi}(\mathbf{t})=\sum_{\mathbf{n}} \mathbf{h}(\mathbf{n}) \sqrt{\mathbf{2}} \boldsymbol{\varphi}(\mathbf{2 t}-\mathbf{n}), \quad \mathbf{n} \in \mathbf{Z}
$$

Where the $\mathrm{h}(\mathrm{n})$ is scaling function. The factor $\sqrt{\mathbf{2}}$ used for normalization of the scaling function. The important feature of signal expressed in terms of wavelet function $\boldsymbol{\psi}_{\mathbf{j}, \mathbf{k}}(\mathbf{t})$ not in scaling function $\boldsymbol{\varphi}_{\mathrm{j}, \mathrm{k}}(\mathbf{t})$. The orthogonal complement of $\mathbf{v}_{\mathbf{j}}$ in $\mathbf{v}_{\mathbf{j}+\mathbf{1}}$ is defined as $\mathrm{W}_{\mathbf{j}}$, we require,

$$
\left\langle\boldsymbol{\varphi}_{\mathrm{j}, \mathrm{k}}(\mathrm{t}) \boldsymbol{\Psi}_{\mathrm{j}, \mathrm{l}}(\mathrm{t})\right\rangle=\int \boldsymbol{\varphi}_{\mathrm{j}, \mathrm{k}}(\mathrm{t}) \boldsymbol{\Psi}_{\mathrm{j}, \mathrm{l}}(\mathrm{t}) \mathrm{dt}
$$

For all appropriate $\mathbf{j}, \mathbf{k}, \mathbf{l} \in \mathbf{Z}$. The relationship of the various subspaces is $\mathbf{v}_{\mathbf{0}} \subset \mathbf{v}_{\mathbf{1}} \subset \mathbf{v}_{\mathbf{2}} \subset \cdots, \subset \mathbf{L}^{2}$. The wavelet spanned subspaces $\mathbf{W}_{\mathbf{0}}$ such that $\mathbf{v}_{\mathbf{1}}=\mathbf{v}_{\mathbf{0}} \otimes \mathbf{w}_{\mathbf{0}}$, which extends to $\mathbf{v}_{\mathbf{2}}=\mathbf{v}_{\mathbf{0}} \otimes \mathbf{w}_{\mathbf{0}} \otimes \mathbf{w}_{\mathbf{1}}$.In general this $\mathbf{L}^{2}=\mathbf{v}_{\mathbf{0}} \otimes \mathbf{w}_{\mathbf{0}} \otimes \mathbf{w}_{\mathbf{1}} \otimes \ldots$ where $\mathbf{v}_{\mathbf{0}}$ is in the space spanned by the scaling function $\boldsymbol{\varphi}(\mathbf{t}-\mathbf{k})$, at $\mathbf{j}=-\infty$, equation becomes

$$
\mathbf{L}^{2}=\ldots \ldots \otimes \mathbf{w}_{-2} \otimes \mathbf{w}_{-1} \otimes \mathbf{w}_{\mathbf{0}} \otimes \mathbf{w}_{\mathbf{1}} \otimes \mathbf{w}_{2} \ldots
$$

eliminating the scaling space altogether. The wavelet can be represented by a weighted sum of shifted scaling function $\boldsymbol{\varphi}(\mathbf{2 t})$ as,

$$
\boldsymbol{\psi}(\mathbf{t})=\sum_{\mathbf{n}} \mathbf{h}_{\mathbf{1}}(\mathbf{n}) \sqrt{\mathbf{2}} \boldsymbol{\varphi}(\mathbf{2 t}-\mathbf{n}), \quad \mathbf{n} \in \mathbf{Z}
$$

For some set of coefficient $\mathbf{h}_{\mathbf{1}}(\mathbf{n})$, this function gives the prototype or mother wavelet $\boldsymbol{\psi}(\mathbf{t})$ for a class of expansion function of the form,

$$
\boldsymbol{\Psi}_{\mathrm{j}, \mathrm{k}}(\mathrm{t})=2^{\mathrm{j} / 2} \boldsymbol{\psi}\left(2^{\mathrm{j}} \mathrm{t}-\mathrm{k}\right)
$$

Where $2^{\mathbf{j}}$ the scaling of $\mathrm{t}$ is, $\mathbf{2}^{-\mathbf{j}} \mathbf{k}$ is the translation in $\mathrm{t}$, and $2^{\mathbf{j} / \mathbf{2}}$ maintains the $\mathbf{L}^{2}$ norms of the wavelet at different scales. The construction of wavelet using set of scaling function $\varphi_{\mathbf{k}}(\mathbf{t})$ and $\boldsymbol{\psi}_{\mathrm{j}, \mathrm{k}}(\mathbf{t})$ that could span all of $\mathbf{L}^{2}(\mathbf{R})$, therefore function $g(t) \in L^{2}(R)$ can be written as

$$
\mathbf{g}(\mathbf{t})=\sum_{\mathbf{k}=-\infty}^{\infty} \mathbf{c}(\mathbf{k}) \boldsymbol{\varphi}_{\mathbf{k}}(\mathbf{t})+\sum_{\mathbf{j}=\mathbf{0}}^{\infty} \sum_{\mathbf{k}=-\infty}^{\infty} \mathbf{d}(\mathbf{j}, \mathbf{k}) \boldsymbol{\Psi}_{\mathbf{j}, \mathbf{k}}(\mathbf{t})
$$

First summation in above equation gives a function that is low resolution of $g(t)$, for each increasing index $j$ in the second summation, a higher resolution function is added which gives increasing details. The function $\mathrm{d}(\mathrm{j}, \mathrm{k})$ indicates the differences between the translation index $\mathrm{k}$, and the scale parameter $\mathrm{j}$. In wavelet analysis expand coefficient at a lower scale level to higher scale level, from equation (10), we scale and translate the time variable to given as

$$
\boldsymbol{\varphi}\left(2^{\mathbf{j}} \mathbf{t}-\mathbf{k}\right)=\sum_{\mathbf{n}} \mathbf{h}(\mathbf{n}) \sqrt{\mathbf{2}} \boldsymbol{\varphi}\left(\mathbf{2}^{\mathbf{j}+\mathbf{1}} \mathbf{t}-\mathbf{2 k}-\mathbf{n}\right) .
$$

After changing variables $m=2 k+n$, above equation becomes

$$
\boldsymbol{\varphi}\left(2^{\mathbf{j}} \mathbf{t}-\mathbf{k}\right)=\sum_{\mathbf{m}} \mathbf{h}(\mathbf{m}-2 \mathbf{k}) \sqrt{2} \boldsymbol{\varphi}\left(2^{\mathbf{j}+1} \mathbf{t}-\mathbf{m}\right) .
$$

If we denote $v_{j}$ as $\mathbf{v}_{\mathbf{j}}={ }_{\mathbf{k}}^{\overline{\operatorname{Span}}_{\mathbf{j}}}\left\{2^{\mathbf{j}} \boldsymbol{\varphi}\left(\mathbf{2}^{\mathbf{j}} \mathbf{t}-\mathbf{k}\right)\right\}$ then $\mathbf{f}(\mathbf{t}) \in$ $\mathbf{v}_{\mathbf{j}+\mathbf{1}} \Rightarrow \mathbf{f}(\mathbf{t})=\sum_{\mathbf{k}} \mathbf{c}_{\mathbf{j}+\mathbf{1}}(\mathbf{k}) \mathbf{2}^{\mathbf{j}+\mathbf{1}} \boldsymbol{\varphi}\left(\mathbf{2}^{\mathbf{j}+\mathbf{1}} \mathbf{t}-\mathbf{k}\right)$. is expressible at scale $\mathrm{j}+1$, with a scaling function only not wavelets. At one scale lower resolution, wavelets are necessary for the detail not available at a scale of $\mathrm{j}$. We have

$$
\mathbf{f}(\mathbf{t})=\sum_{\mathbf{k}} \mathbf{c}_{\mathbf{j}}(\mathbf{k}) \mathbf{2}^{\mathrm{j} / \mathbf{2}} \boldsymbol{\varphi}\left(2^{\mathrm{j}} \mathbf{t}-\mathbf{k}\right)+\sum_{\mathrm{k}} \mathbf{d}_{\mathrm{j}}(\mathbf{k}) \mathbf{2}^{\frac{\mathrm{j}}{2}} \boldsymbol{\varphi}\left(2^{\mathrm{j}} \mathbf{t}-\mathbf{k}\right) .(15)
$$

Where the $2^{j}$ terms maintain the unity norm of the basis functions at various scales. If $\varphi_{\mathrm{j}, \mathrm{k}}(\mathrm{t})$ and $\psi_{\mathrm{j}, \mathrm{k}}(\mathrm{t})$ are orthonormal, the $\mathrm{j}$ level scaling coefficients are found by taking the inner product

$$
\mathbf{c}_{\mathbf{j}}(\mathbf{k})=\left\langle\mathbf{f}(\mathbf{t}), \boldsymbol{\varphi}_{\mathrm{j}, \mathrm{k}}(\mathrm{t})\right\rangle=\int \mathbf{f}(\mathrm{t}) 2^{\mathrm{j} / 2} \boldsymbol{\varphi}\left(2^{\mathrm{j}} \mathbf{t}-\mathbf{k}\right) \mathbf{d t} .
$$

By using equation (14) and interchanging the sum and integral, can be written as

$$
\mathbf{c}_{\mathbf{j}}(\mathbf{k})=\sum_{\mathbf{m}} \mathbf{h}(\mathbf{m}-\mathbf{2 k}) \int \mathbf{f}(\mathbf{t}) \mathbf{2}^{\frac{\mathbf{j}+\mathbf{1}}{2}} \boldsymbol{\varphi}\left(\mathbf{2}^{\mathbf{j}+\mathbf{1}} \mathbf{t}-\mathbf{m}\right) \mathbf{d t} .
$$

But the integral is inner product with the scaling function at a scale $\mathrm{j}+1$ giving

$$
\mathbf{c}_{\mathbf{j}}(\mathbf{k})=\sum_{\mathbf{m}} \mathbf{h}(\mathbf{m}-\mathbf{2 k}) \mathbf{c}_{\mathbf{j}+\mathbf{1}}(\mathbf{m}) .
$$

The corresponding wavelet coefficient is

$$
\mathbf{d}_{\mathbf{j}}(\mathbf{k})=\sum_{\mathbf{m}} \mathbf{h}_{\mathbf{1}}(\mathbf{m}-\mathbf{2 k}) \mathbf{c}_{\mathbf{j}+\mathbf{1}}(\mathbf{m}) \text {. }
$$

Fig. 2 shows the structure of two stages down sampling filter banks in terms of coefficients.

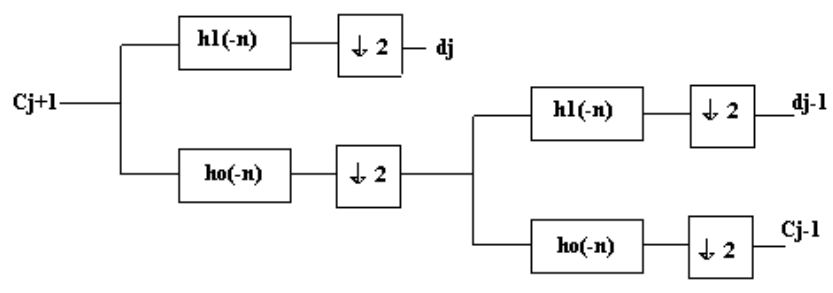

Figure 2: Two stages down sampling filter bank

A reconstruction of the original fine scale coefficient of the signal made from a combination of the scaling function and wavelet coefficient at a course resolution which is derived by 
considering a signal in the $\mathbf{j}+\mathbf{1}$ scaling function space $\mathbf{f}(\mathbf{t}) \in$ $\mathbf{v}_{\mathbf{j}+\mathbf{1}}$. This function written in terms of the scaling function as

$$
\mathbf{f}(\mathbf{t})=\sum_{\mathrm{k}} \mathbf{c}_{\mathbf{j}+\mathbf{1}}(\mathbf{k}) 2^{\frac{\mathrm{j}+1}{2}} \boldsymbol{\varphi}\left(2^{\mathrm{j}+1} \mathbf{t}-\mathbf{k}\right) .
$$

In terms of next scales which requires wavelet as

$$
\mathbf{f}(\mathbf{t})=\sum_{\mathbf{k}} \mathbf{c}_{\mathbf{j}}(\mathbf{k}) 2^{\mathbf{j} / 2} \boldsymbol{\varphi}\left(2^{\mathbf{j}} \mathbf{t}-\mathbf{k}\right)+\sum_{\mathbf{k}} \mathbf{d}_{\mathbf{j}}(\mathbf{k}) 2^{\mathbf{j}} \boldsymbol{\Psi}\left(2^{\mathbf{j}} \mathbf{t}-\mathbf{k}\right)
$$

Substituting equation (15) and equation (11) into equation (20), gives

$$
\begin{array}{r}
\mathbf{f}(\mathbf{t})=\sum_{\mathbf{k}} \mathbf{c}_{\mathbf{j}}(\mathbf{k}) \sum_{\mathbf{n}} \mathbf{h}(\mathbf{n}) \mathbf{2}^{\frac{\mathrm{j}+\mathbf{1}}{2}} \boldsymbol{\varphi}\left(2^{\mathbf{j}+1} \mathbf{t}-\mathbf{2 k}-\mathbf{n}\right)+ \\
\sum_{\mathbf{k}} \mathbf{d}_{\mathbf{j}}(\mathbf{k}) \sum_{\mathbf{n}} \mathbf{h}_{\mathbf{1}}(\mathbf{n}) 2^{\frac{\mathrm{j}+1}{2}} \boldsymbol{\varphi}\left(2^{\mathbf{j}+1} \mathbf{t}-\mathbf{2 k}-\mathbf{n}\right) .
\end{array}
$$

Because all of these function are orthonormal, multiplying

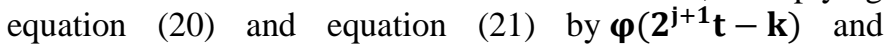
integrating evaluates the coefficients as

$$
\begin{aligned}
\mathbf{c}_{\mathbf{j}+\mathbf{1}}(\mathbf{k})= & \sum_{\mathbf{m}} \mathbf{c}_{\mathbf{j}}(\mathbf{m}) \mathbf{h}(\mathbf{k}-\mathbf{2 m})+ \\
& \sum_{\mathbf{m}} \mathbf{d}_{\mathbf{j}}(\mathbf{m}) \mathbf{h}_{\mathbf{1}}(\mathbf{k}-\mathbf{2 m}) .
\end{aligned}
$$

Fig. 3 shows the structure of two stages up sampling filter banks in terms of coefficients i.e. synthesis from coarse scale to fine scale [5] [6] [7].

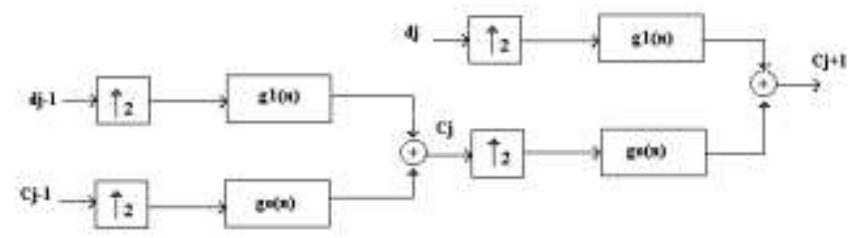

Figure 3: Two stages up sampling filter

In filter structure analysis can be done by apply one step of the one dimensional transform to all rows, then repeat the same for all columns then proceed with the coefficients that result from a convolution with in both directions[6][7][8][9][10][12]. The two level wavelet decomposition as shown in fig 4 .
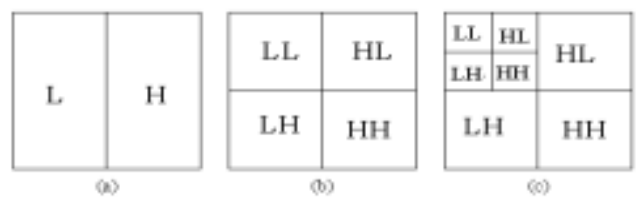

Figure 4: Two-dimensional wavelet transform.

\section{Denoising Technique With Existing Threshold}

Noise is present in an image either in an additive or multiplicative form. An additive noise follows the rule, $\mathbf{w}(\mathbf{x}, \mathbf{y})=\mathbf{s}(\mathbf{x}, \mathbf{y})+\mathbf{n}(\mathbf{x}, \mathbf{y})$

While the multiplicative noise satisfies

$$
\mathbf{w}(\mathbf{x}, \mathbf{y})=\mathbf{s}(\mathbf{x}, \mathbf{y}) \times \mathbf{n}(\mathbf{x}, \mathbf{y}) .
$$

Where $\mathbf{s}(\mathbf{x}, \mathbf{y})$ is the original signal, $\mathbf{n}(\mathbf{x}, \mathbf{y})$ denotes the noise. When noise introduced into the signal it produces the corrupted image $\mathbf{w}(\mathbf{x}, \mathbf{y}) .14]$. Gaussian Noise is evenly distributed over the signal. This means that each pixel in the noisy image is the sum of the true pixel value and a random
Gaussian distributed noise value. Salt and Pepper Noise is an impulse type of noise, which is also referred to as intensity spikes. This is caused generally due to errors in data transmission. The corrupted pixels are set alternatively to the minimum or to the maximum value, giving the image a "salt and pepper" like appearance. Unaffected pixels remain unchanged. The source of this noise is attributed to random interference between the coherent returns [7], [8], [9] [10]. Fully developed speckle noise has the characteristic of multiplicative noise.

\section{A. Universal Threshold}

The universal threshold can be defined as,

$$
\mathrm{T}=\boldsymbol{\sigma} \sqrt{\operatorname{2\operatorname {log}(\mathrm {N})}}
$$

$\mathrm{N}$ being the signal length, $\boldsymbol{\sigma}$ being the noise variance is well known in wavelet literature as the Universal threshold. It is the optimal threshold in the asymptotic sense and minimizes the cost function of the difference between the function. One can surmise that the universal threshold may give a better estimate for the soft threshold if the number of samples is large [13] [14].

\section{B. Visu Shrink}

Visu Shrink was introduced by Donoho [13]. It uses a threshold value $t$ that is proportional to the standard deviation of the noise. It follows the hard threshold rule. An estimate of the noise level $\sigma$ was defined based on the median absolute deviation given by

$$
\widehat{\boldsymbol{\sigma}}=\frac{\operatorname{Median}\left(\left\{\left|\mathbf{g}_{\mathbf{j}-1, \mathbf{k}}\right|: \mathbf{k}=0,1, \ldots . \mathbf{2}^{\mathbf{j}-1}-\mathbf{1}\right\}\right)}{0.6745}
$$

Whereg $_{j-1, k}$ corresponds to the detail coefficients in the wavelet transform. VisuShrink does not deal with minimizing the mean squared error. Another disadvantage is that it cannot remove speckle noise. It can only deal with an additive noise. VisuShrink follows the global threshold scheme, which is globally to all the wavelet coefficients [9].

\section{Sure Shrink}

A threshold chooser based on Stein's Unbiased Risk Estimator (SURE) was proposed by Donoho and Johnstone and is called as Sure Shrink. It is a combination of the universal threshold and the SURE threshold [15] [16]. This method specifies a threshold value $\mathbf{t}_{\mathbf{j}}$ for each resolution level $\mathbf{j}$ in the wavelet transform which is referred to as level dependent threshold. The goal of Sure Shrink is to minimize the mean squared error [9], defined as,

$$
\operatorname{MSE}=\frac{1}{n^{2}} \sum_{x, y-1}^{n}(Z(x, y)-S(x, y))^{2}
$$

Where $\mathbf{z}(\mathbf{x}, \mathbf{y})$ is the estimate of the signal, $\mathbf{s}(\mathbf{x}, \mathbf{y})$ is the original signal without noise and $\mathrm{n}$ is the size of the signal. Sure Shrink suppresses noise by threshold the empirical wavelet coefficients. The Sure Shrink threshold t* is defined as

$$
\mathbf{t}^{*}=\min (\mathbf{t}, \sigma \sqrt{2 \log \mathbf{n}})
$$

Where $t$ denotes the value that minimizes Stein's Unbiased Risk Estimator, $\sigma$ is the noise variance computed from 
Equation, and $\mathrm{n}$ is the size of the image. It is smoothness adaptive, which means that if the unknown function contains abrupt changes or boundaries in the image, the reconstructed image also does [17] [18].

\section{Bayes Shrink}

Bayes Shrink was proposed by Chang, Yu and Vetterli. The goal of this method is to minimize the Bayesian risk, and hence its name, Bayes Shrink [19]. The Bayes threshold, $t_{B}$, is defined as

$$
\mathbf{t}_{\mathrm{B}}=\boldsymbol{\sigma}^{2} / \boldsymbol{\sigma}_{\mathrm{s}}
$$

Where $\sigma^{2}$ is the noise variance and $\sigma_{\mathrm{s}}$ is the signal variance without noise. The noise variance $\sigma^{2}$ is estimated from the sub band $\mathrm{HH}$ by the median estimator shown in equation (28). From the definition of additive noise we have $\mathbf{w}(\mathbf{x}, \mathbf{y})=\mathbf{s}(\mathbf{x}, \mathbf{y})+\mathbf{n}(\mathbf{x}, \mathbf{y})$. Since the noise and the signal are independent of each other, it can be stated that

$$
\begin{aligned}
& \boldsymbol{\sigma}_{\mathrm{w}}^{2}=\boldsymbol{\sigma}_{\mathrm{s}}^{2}+\boldsymbol{\sigma}^{2} . \\
& \quad \sigma_{\mathrm{w}}^{2} \text { can be computed as shown below: } \\
& \boldsymbol{\sigma}_{\mathbf{w}}^{2}=\frac{1}{\mathbf{n}^{2}} \sum_{\mathbf{x}, \mathbf{y}-\mathbf{1}}^{\mathbf{1}} \mathbf{w}^{2}(\mathbf{x}, \mathbf{y}) .
\end{aligned}
$$

The variance of the signal, $\sigma^{2}$ is computed as

$$
\sigma_{\mathrm{s}}=\sqrt{\max \left(\sigma_{\mathrm{w}}^{2}-\sigma^{2}, \mathbf{0}\right)}
$$

With $\sigma^{2}$ and $\sigma_{s}^{2}$, the Bayes threshold is computed from Equation (31). Using this threshold, the wavelet coefficients are threshold at each band [20].

\section{E. Normal Shrink:}

The threshold value which is adaptive to different sub band characteristics

$$
\mathrm{TN}=\boldsymbol{\beta}^{\boldsymbol{\sigma}^{2}} / \sigma_{\mathrm{y}}
$$

Where the scale parameter $\beta$ has computed once for each scale, using the following equation.

$$
\beta=\sqrt{\log \left(\frac{\mathrm{L}_{\mathrm{k}}}{\mathrm{J}}\right)} .
$$

$\mathrm{L}_{\mathrm{k}}$ means the length of the sub band at $\mathbf{K}^{\text {th }}$ scale. $\boldsymbol{\sigma}^{2}$ means the noise variance, [13] which can be estimated from the sub band $\mathrm{HH}$ using equation (32).

\section{Proposed Denoising SCHEME}

There are different denoising scheme used to remove noise while preserving original information and basic parameter of the image. Contrast, brightness, edges and background of the image should be preserved while denoising in this technique. Wavelet transform tool used in denoising of image. Multi resolution analysis structure consider for denoising scheme. Actually, the performance of our algorithm is very close, and in some cases even surpasses, to that of the already published denoising methods. Performance measured in terms of signal to noise ratio

\section{A. New threshold function:}

This function is calculated by

$$
\text { newth }=\sqrt{2 \mathrm{~m} \times \log (M)}
$$

Where, $\mathbf{M}$ is the total number of pixel of an image, $\mathbf{m}$ is the mean of the image. This function preserves the contrast, edges, background of the images. This threshold function calculated at different scale level.

\section{B. Circular kernel:}

Kernel applied to the wavelet approximation coefficient, to get denoised image with all parameters undisturbed. The kernel uses here in this technique contains some components like [0 $0111100 ; 01111110 ; 11111111$; 111111111 ; 1

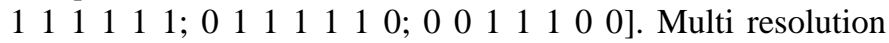
analysis wavelet structure has used for this kernel to get result.

\section{Mean-Max threshold:}

Generation of the threshold in using mean and max method after decomposition. Let xi denotes the sequence of elements; threshold can be calculated using following technique.

$$
\begin{aligned}
& \operatorname{MAXMIN}=\left\{\mathbf{x}_{\mathbf{j}}\right\}=\operatorname{MAX}\left\{\left[\operatorname{MIN}\left(\mathbf{x}_{1}, \ldots \ldots \mathbf{x}_{\mathbf{k}}\right],\right.\right. \\
& \left.\left[\operatorname{MIN}\left(\mathrm{x}_{2}, \ldots \ldots \mathrm{x}_{\mathrm{k}+1}\right)\right], \ldots \ldots+\left[\operatorname{MIN}\left(\mathrm{x}_{\mathbf{i}-\mathrm{k}+1}, \ldots \ldots \mathrm{x}_{\mathbf{i}}\right)\right]\right\} \\
& \operatorname{MINMAX}=\left\{\mathbf{x}_{\mathbf{j}}\right\}=\operatorname{MIN}\left\{\left[\operatorname{MAX}\left(\mathrm{x}_{1}, \ldots \ldots \mathbf{x}_{\mathbf{k}}\right]\right.\right. \text {, } \\
& \left.\left[\operatorname{MAX}\left(\mathbf{x}_{2}, \ldots . . \mathbf{x}_{\mathbf{k}+1}\right)\right], \ldots \ldots+\left[\operatorname{MAX}\left(\mathbf{x}_{\mathbf{i}-\mathbf{k}+1}, \ldots \ldots \mathbf{x}_{\mathbf{i}}\right)\right]\right\}
\end{aligned}
$$

\section{Nearest neighbor:}

This technique gives better result for different kernel structure shown in figure (5). In this kernel central pixel (CP), calculated from the neighbor value. Three different kernels have proposed for better reduction of noise using wavelet transform at different scale. Mark ' $x$ ' denotes low value at that position.

\begin{tabular}{|c|c|c|}
\hline 1 & $\mathrm{X}$ & 2 \\
\hline $\mathrm{X}$ & $\mathrm{CP}$ & $\mathrm{x}$ \\
\hline 4 & $\mathrm{X}$ & 3 \\
\hline
\end{tabular}

(a)

\begin{tabular}{|c|c|c|c|c|c|}
\hline $\mathrm{X}$ & 1 & $\mathrm{X}$ & 1 & 2 & 3 \\
\hline 2 & $\mathrm{CP}$ & 4 \\
\hline $\mathrm{X}$ & 3 & $\mathrm{X}$ & $\mathrm{CP}$ & 5 \\
\hline 6 & 7 & 8 \\
\hline
\end{tabular}

(b) (c)
Figure 5: Kernel at different noise level.

\section{RESUlT}

Image parameters has not disturb when denoising. In this paper calculating threshold function in spatial domain, and Lena image is used for implementation. When denoising we have to preserve contrast of the image. Image brightness in denoising kept same but preserves the background and the gray level tonalities in the image. The noise term is considered as a random phenomenon and it is uncorrelated, hence the average value of the noise results in a zero value, therefore consider proper kernel to get denoised image The low pass spatial filter reduces the noise such as bridging the gaps in the lines or curve in a given image, but not suitable for reducing the noise patterns consisting of strong spike like components [21] [22]. The high pass filters results in sharp details, it provides more visible details that obscured, hazy, and poor focus on the original image. Now wavelets preferred in denoising while preserving all the details of the image. Table 1 shows the results with existing technique and proposed denoising scheme. 
TABLE 1: RESULT OF DIFFERENT TECHNIQUE WITH LENA.

\begin{tabular}{|c|c|c|c|c|c|}
\hline \multicolumn{2}{|c|}{ Methods } & \multicolumn{4}{|c|}{ Denoised image SNR } \\
\hline & & Gaussian & $\begin{array}{r}\text { Salt and } \\
\text { Pepper }\end{array}$ & Poisson & Speckle \\
\hline \multicolumn{2}{|c|}{ VisuShrink } & 14.2110 & 11.8957 & 11.6988 & 12.2023 \\
\hline \multicolumn{2}{|c|}{ Universal } & 9.2979 & 9.6062 & 9.5227 & 9.8297 \\
\hline \multicolumn{2}{|c|}{ Sure shrink } & 16.8720 & 17.2951 & 14.3082 & 14.7922 \\
\hline \multicolumn{2}{|c|}{ Normal Shrink } & 15.4684 & 17.2498 & 14.5519 & 15.0069 \\
\hline \multicolumn{2}{|c|}{ Bays shrink } & 15.3220 & 16.9775 & 14.6518 & 14.9716 \\
\hline \multicolumn{2}{|c|}{ New threshold } & 15.0061 & 15.5274 & 13.9969 & 14.5171 \\
\hline \multicolumn{2}{|c|}{ Circular kernel } & 16.7560 & 18.9791 & 17.9646 & 14.2433 \\
\hline \multirow{5}{*}{$\begin{array}{c}\text { Mean } \\
\text { Max } \\
\text { approxim } \\
\text { ation }\end{array}$} & Maxmin & 9.5908 & 10.2514 & 10.0308 & 10.4287 \\
\hline & Meanmin & 12.5421 & 12.9643 & 13.2045 & 13.3588 \\
\hline & Minmax & 13.3761 & 9.3629 & 13.4904 & 13.8338 \\
\hline & Meanmax & 10.7183 & 11.5483 & 11.3422 & 11.8212 \\
\hline & Sqrtth & 16.9222 & 19.9917 & 16.9681 & 16.4639 \\
\hline \multirow{3}{*}{$\begin{array}{l}\text { Nearest } \\
\text { Neighbor }\end{array}$} & $\begin{array}{c}\text { Four } \\
\text { diagonal }\end{array}$ & 13.6178 & 13.4029 & 12.9661 & 13.6229 \\
\hline & $\begin{array}{c}\text { Four } \\
\text { directional }\end{array}$ & 13.7226 & 13.1139 & 13.1241 & 13.8448 \\
\hline & $\begin{array}{c}\text { Eight conn- } \\
\text { ectivity }\end{array}$ & 13.5136 & 13.0216 & 13.0835 & 13.9546 \\
\hline
\end{tabular}

\section{CONCLUSION}

This technique is computationally faster and gives better results. Some aspects that were analyzed in this paper may be useful for other denoising schemes, objective criteria for evaluating noise suppression performance of different significance measures. Our new threshold function is better as compare to other threshold function. Some function gives better edge perseverance, background information, contrast stretching, in spatial domain. In future we can use same threshold function for medical images as well as texture images to get denoised image with improved performance parameter.

\section{REFERENCES}

[1] Donoho.D.L,Johnstone.I.M, "Ideal spatial adaptation via wavelet shrinkage", Biometrika,81,pp.425-455,1994.

[2] Gao Zhing, Yu Xiaohai, "Theory and application of MATLAB Wavelet analysis tools", National defense industry publisher,Beijing,pp.108-116, 2004.

[3] Aglika Gyaourova Undecimated wavelet transforms for image denoising, November 19, 2002.

[4] Bart Goossens, Aleksandra Pi`zurica, and Wilfried Philips, "Image Denoising Using Mixtures of Projected Gaussian Scale Mixtures", IEEE
Transactions On Image Processing, Vol. 18, No. 8, August 2009, Pp. 1689-1702

[5] Michel Misiti, Yves Misiti, Georges Oppenheim, Jean-Michel Poggi, "Wavelets and their Applications", Published by ISTE 2007 UK.

[6] C Sidney Burrus, Ramesh A Gopinath, and Haitao Guo, "Introduction to wavelet and wavelet transforms", Prentice Hall1997.S. Mallat, A Wavelet Tour of Signal Processing, Academic, New York, second edition, 1999.

[7] R. C. Gonzalez and R. Elwood's, Digital Image Processing. Reading, MA: Addison-Wesley, 1993.

[8] M. Sonka,V. Hlavac, R. Boyle Image Processing , Analysis , And Machine Vision. Pp10-210 \& 646-670

[9] Raghuveer M. Rao., A.S. Bopardikar Wavelet Transforms: Introduction To Theory And Application Published By Addison-Wesley 2001 pp1126

[10] Arthur Jr Weeks, Fundamental of Electronic Image Processing PHI 2005.

[11] Jaideva Goswami Andrew K. Chan, "Fundamentals Of Wavelets Theory, Algorithms, And Applications", John Wiley Sons

[12] Donoho, D.L. and Johnstone, I.M. (1994) Ideal spatial adaptation via wavelet shrinkage. Biometrika, 81, 425-455.

[13] D. L. Donoho and I. M. Johnstone, "Denoising by soft thresholding", IEEE Trans. on Inform. Theory, Vol. 41, pp. 613-627, 1995.

[14] Mark J. T. Smith and Steven L. Eddins, "Analysis/synthesis techniques for sub band image coding,"IEEE Trans. Acoust., Speech and Signal Process., vol. 38,no. 8, pp. 1446-1456, Aug. 1990.

[15] F. Luisier, T. Blu, and M. Unser, "A new SURE approach to image denoising: Inter-scale orthonormal wavelet thresholding," IEEE Trans. Image Process., vol. 16, no. 3, pp. 593-606, Mar. 2007.

[16] X.-P. Zhang and M. D. Desai, "Adaptive denoising based on SURE risk,” IEEE Signal Process. Lett., vol. 5, no. 10, pp. 265-267, Oct. 1998.

[17] Thierry Blu, and Florian Luisier "The SURE-LET Approach to Image Denoising". IEEE Transactions On Image Processing, VOL. 16, NO. 11, pp $2778-2786$, NOV 2007 .

[18] H. A. Chipman, E. D. Kolaczyk, and R. E. McCulloch: 'Adaptive Bayesian wavelet shrinkage', J. Amer. Stat. Assoc., Vol. 92, No 440, Dec. 1997, pp. 1413-1421

[19] Chang, S. G., Yu, B., and Vetterli, M. (2000). Adaptive wavelet thresholding for image denoising and compression. IEEE Trans. on Image Proc., 9, 1532-1546.

[20] Andrea Polesel, Giovanni Ramponi, And V. John Mathews, "Image Enhancement Via Adaptive Unsharp Masking" IEEE Transactions On Image Processing, Vol. 9, No. 3, March 2000, Pp505-509

[21] G. Y. Chen, T. D. Bui And A. Krzyzak, Image Denoising Using Neighbouringwavelet Coefficients, Icassp ,Pp917-920

[22] Sasikala, P. (2010). Robust R Peak and QRS detection in Electrocardiogram using Wavelet Transform. International Journal of Advanced Computer Science and Applications - IJACSA, 1(6), 48-53.

[23] Kekre, H. B. (2011). Sectorization of Full Kekre's Wavelet Transform for Feature extraction of Color Images. International Journal of Advanced Computer Science and Applications - IJACSA, 2(2), 69-74.

\section{AUTHORS PROFILE}

Ruikar Sachin D has received the postgraduate degree in Electronics and Telecommunication Engineering from Govt Engg College, Pune University, India in 2002. He is currently pursuing the Ph.D. degree in Electronics Engineering, SGGS IET, SRTMU Nanded, India. His research interests include image denoising with wavelet transforms, image fusion and image in painting.

Dharmpal D Doye received his BE (Electronics) degree in 1988, ME (Electronics) degree in 1993 and Ph. D. in 2003 from SGGS College of Engineering and Technology, Vishnupuri, Nanded (MS) - INDIA. Presently, he is working as Professor in department of Electronics and Telecommunication Engineering, SGGS Institute of Engineering and Technology, Vishnupuri, Nanded. His research fields are speech processing, fuzzy neural networks and image processing. 